NIST Technical Note 1991

\title{
Investigations on SI-Traceable Dynamic Calibration of Instrumented Charpy Strikers
}

\author{
Akobuije Chijioke \\ Nicholas Vlajic \\ Edward Mulhern \\ Enrico Lucon
}

This publication is available free of charge from:

https://doi.org/10.6028/NIST.TN.1991

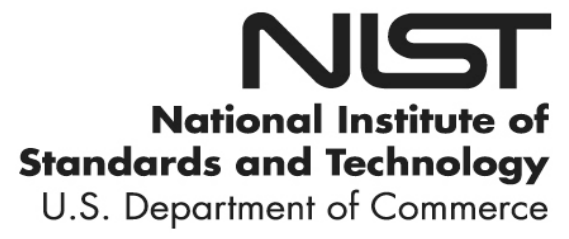




\title{
Investigations on SI-Traceable Dynamic Calibration of Instrumented Charpy Strikers
}

\author{
Akobuije Chijioke \\ Nicholas Vlajic \\ Edward Mulhern \\ Quantum Measurement Division \\ Physical Measurement Laboratory \\ Enrico Lucon \\ Applied Chemicals and Materials Division \\ Material Measurement Laboratory
}

This publication is available free of charge from:

https://doi.org/10.6028/NIST.TN.1991

May 2018

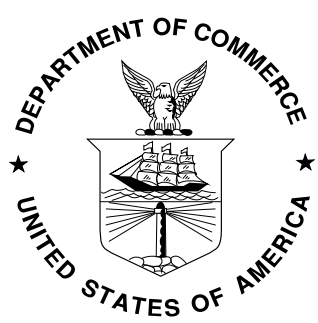

U.S. Department of Commerce

Wilbur L. Ross, Jr., Secretary

National Institute of Standards and Technology Walter Copan, NIST Director and Undersecretary of Commerce for Standards and Technology 
Certain commercial entities, equipment, or materials may be identified in this document in order to describe an experimental procedure or concept adequately. Such identification is not intended to imply recommendation or endorsement by the National Institute of Standards and Technology, nor is it intended to imply that the entities, materials, or equipment are necessarily the best available for the purpose.

National Institute of Standards and Technology Technical Note 1991

Natl. Inst. Stand. Technol. Tech. Note 1991, 31 pages (May 2018) CODEN: NTNOEF

This publication is available free of charge from:

https://doi.org/10.6028/NIST.TN.1991 


\begin{abstract}
In this Technical Note, we report on investigations toward an SI-traceable dynamic calibration of instrumented Charpy strikers, performed at the National Institute of Standards and Technology (NIST) from January to December 2017. Although Charpy tests are fewmillisecond-duration events in which dynamic phenomena are often clearly present, the force measurements from instrumented Charpy tests are predominantly obtained using static calibrations, which has long been recognized as a source of significant potential errors. We discuss a number of approaches to dynamic calibration, including methods that have previously been implemented at NIST (and elsewhere), and methods that are new in the sense that they are not presently commonly used or incorporated into standards. We describe exploratory tests of some of the new calibration approaches, the results of these tests, and how the information obtained is being applied to further work. A key objective of these investigations is that the calibration be traceable to the International System of Units.
\end{abstract}

\title{
Key words
}

Dynamic calibration; instrumented Charpy striker; International System of Units (SI), SI traceability; static calibration. 


\section{Table of Contents}

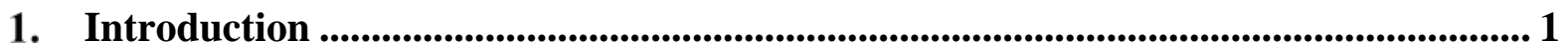

2. Established Methods for Calibrating Instrumented Charpy Strikers ......................... 4

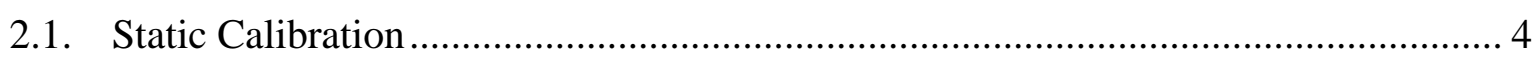

2.2. Energy Adjustment Method ............................................................................. 5

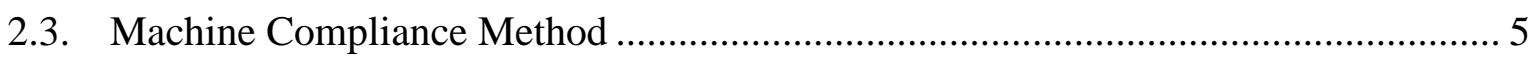

3. Possible Options for an SI-Traceable Dynamic Calibration .......................................... 8

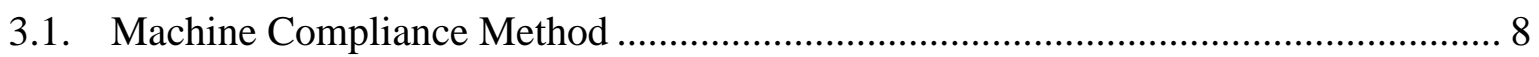

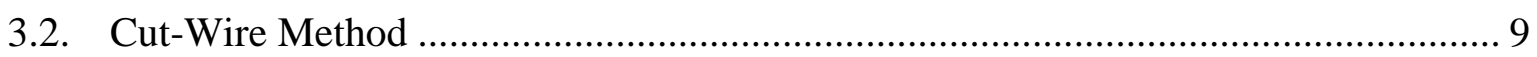

3.3. Mass Impact Method ...................................................................................... 10

3.4. Dynamically Calibrated Transfer Transducer ............................................................ 11

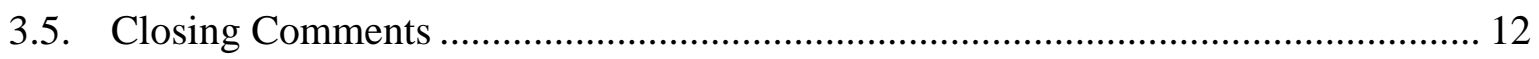

4. Dynamic Calibration Exercise........................................................................................... 13

4.1. Cut-Wire Calibration Test .............................................................................. 13

4.2. Calibrated Transfer Transducer Test ...................................................................... 16

4.3. Summary and Lessons Learned............................................................................ 19

5. Current Efforts for Dynamically Calibrating an Instrumented Charpy Striker.... 21

5.1. Design Modifications to Improve Instrumented Striker Performance ....................... 21

5.2. Extension of Transfer Transducer Calibration Bandwidth and Mechanization of Impacts ........................................................................................................ 22

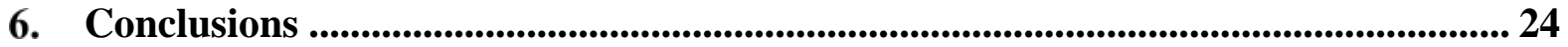

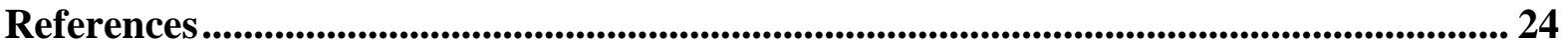

\section{List of Figures}

Fig. 1. Schematic drawing of a Charpy machine and a Charpy specimen being tested (image reproduced and modified from https://commons.wikimedia.org/).

Fig. 2. Example of relationship between applied force and striker output obtained from the static calibration of a NIST instrumented striker. A linear and a quadratic fit are shown in the figure.

Fig. 3. Example of a force-time curve obtained from an elastic low-blow test performed on an unnotched Charpy specimen for the measurement of the compliance of a Charpy machine.

Fig. 4. Cut-wire method with (a) tensile loading and (b) compressive loading; (c) and (d) show idealized generated force steps by configurations (a) and (b) respectively, in arbitrary units.

Fig. 5. Computer rendering of mock Charpy machine used for testing calibration methodologies. .13 
Fig. 6. Photograph rendering of the setup used for testing the cut-wire calibration method.. 14

Fig. 7. Data acquisition arrangement used for testing cut-wire calibration method............... 15

Fig. 8. Voltage trace for an experimental cut-wire calibration test. ..................................... 15

Fig. 9. Experimental calibration spectra for the striker mounted in the mock-Charpy machine. Results are shown for cut-wire and transfer-standard based calibration tests. The error bounds, shown as thin dashed lines for the cut-wire traces and as vertical bars for the impact data, are the scatter (1 standard deviation) of the acquired data............................................... 16

Fig. 10. Typical construction of an impact hammer. ........................................................... 17

Fig. 11. Data acquisition arrangement used for testing the calibrated transfer transducer method.

Fig. 12. Dependence of calibration on person swinging hammer, for striker mounted in mock-Charpy machine. Error bars represent 1 standard deviation.

Fig. 13. Photographs of the (a) instrumented striker that was designed to have an invariant sensitivity to impact location and (b) the strain gage configuration on one side of the instrumented striker.

Fig. 14. Comparison of the normalized sensitivity as a function of impact location for the instrumented striker presented in Section 5 to the one shown in Section 4. 22 Fig. 15. Prototype mechanical device used to secure the transfer standard, which can be traceably calibrated by striking a mass and measuring its displacement. 


\section{Introduction}

Charpy impact testing is an experimental test used worldwide since the early 1900s for characterizing the notch toughness of metals, and thereby their suitability for applications ranging from ship hulls and railroad tracks to nuclear reactor pressure vessels. A standard Charpy test consists of a swinging mass (pendulum) dropping from a known height and striking a notched specimen located in its path; the specimen fractures, and the difference between the height to which the pendulum rises and its original height provides a measurement of the energy absorbed in breaking the specimen. The Charpy verification program at the National Institute of Standards and Technology (NIST) supplies thousands of customers worldwide with verification steel specimens that have been certified on the three NIST reference Charpy machines; this program achieves the tightest consistency of any population of Charpy machines in the world [1]. A schematic drawing of a Charpy machine, which identifies its different parts and shows a specimen being impacted, is provided in Fig. 1.

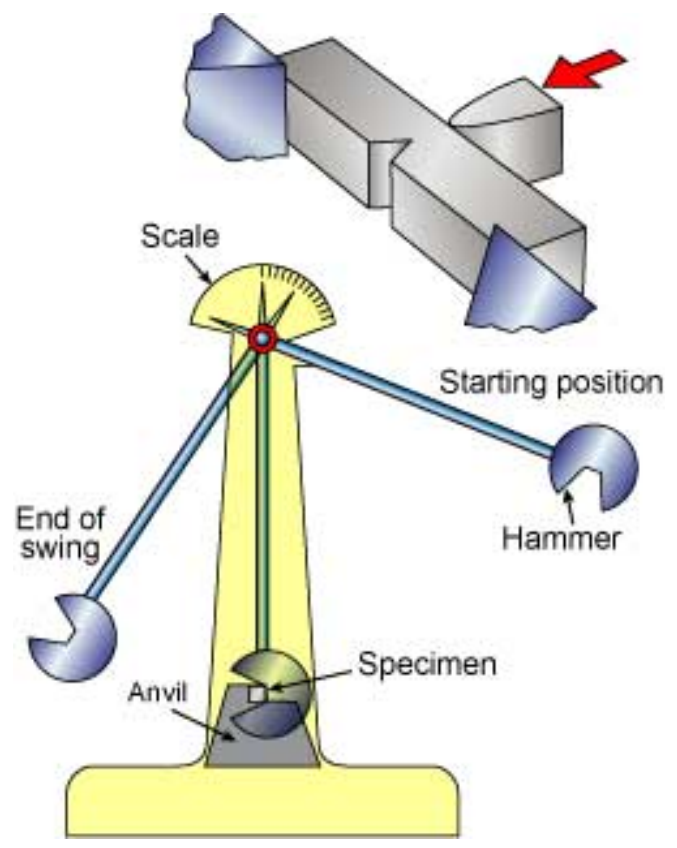

Fig. 1. Schematic drawing of a Charpy machine and a Charpy specimen being tested (image reproduced and modified from https://commons.wikimedia.org/).

In the instrumented version of the Charpy test, a number of strain gauges attached to the striker transform it into a force transducer that provides an indication of the force applied to the specimen during impact. The force-time record derived from an instrumented Charpy striker offers additional information about the tested material's properties in comparison to a standard (i.e., non-instrumented) impact test. However, impact tests are highly dynamic events, with a duration ranging from $0.1 \mathrm{~ms}$ to $5 \mathrm{~ms}$, with force pulse amplitudes typically exceeding $25 \mathrm{kN}$ for steel specimens. Obtaining an accurate SI-traceable calibration for an instrumented Charpy striker has remained elusive. Currently, instrumented impact tests are analyzed based on a static calibration of the instrumented striker. Transforming voltage-time data into force-time data by means of a static calibration is not expected to deliver high accuracy, as the sensitivity of the instrumented striker mounted in the Charpy machine will 
exhibit some degree of dependence on excitation rate (e.g. a non-flat frequency response function). For example, resonances in the response that are excited by the impact event will be falsely interpreted as force oscillation ${ }^{1}$, if the interpretation is based on a static calibration.

Some improvement is achieved by correcting the observed force values by equalizing the absorbed energy derived from the pendulum swing angles and the instrumented energy obtained by integrating force with respect to inferred displacement [3]. This approach has been incorporated into the ASTM E2298 standard [4]. However, as shown in Ref. [3] and discussed below, there is room for improvement. An alternative approach, which aims at exploring higher loading rates, consists in inferring the conversion factor between striker signal and force from a purely elastic, low-blow instrumented impact test of an unnotched Charpy specimen [5]. This test allows determining the compliance of an impact machine [6]. Other efforts to perform an actual dynamic calibration (see e.g. [7, 8]) have not led to lowabsolute-uncertainty force-versus-time data from instrumented Charpy tests. In this Technical Note, we describe an effort to perform a low-uncertainty, SI-traceable, dynamic force calibration of instrumented Charpy strikers at NIST. Success of this effort would represent a major advance, improving interpretation of instrumented test results and potentially resolving current disagreements between results obtained on different machines and by different institutions.

In general, it is not possible to represent the dynamic sensitivity of a Charpy instrumented striker as a single time-independent function of force. Methods of representing the dynamic sensitivity include the frequency response function (frequency domain) or the impulse response function (time domain), which assume linearity and time-invariance ${ }^{2}$ of the striker response. Provided that these conditions are satisfied, such a calibration provides the sensitivity to any applied force trace. Determining such a dynamic sensitivity from a calibration requires the application of a known, sufficiently fast, dynamic force to the striker. Using the determined dynamic sensitivity function to convert the voltage-time trace obtained in breaking a specimen into a force-time trace is an inverse problem, and in general will require regularization in order to provide a meaningful result [9].

For the dynamically calibrated instrumented striker to provide force values of greatest utility, it is important that the calibration, as well as the subsequent use of the calibration to interpret test results, be traceable. Metrological traceability requires an unbroken chain of calibrations to specified references, and allows measurement results to be reported in units of an agreed reference with quantified uncertainties. It is formally defined as the "property of a measurement result whereby the result can be related to a reference through a documented unbroken chain of calibrations, each contributing to the measurement uncertainty" [10]. SI-traceability means that the reference is (a combination of) SI base units. Thus, for the calibration of an instrumented Charpy striker to yield traceable test results in units of Newtons (or any other force unit defined as a fixed number of Newtons):

(a) The force values applied in the calibration of the striker must have a documented chain of evaluation back to SI base quantities such as the meter, kilogram, Ampere and second, with quantified uncertainties at each step of the chain, giving rise to the final uncertainties of the applied force values during the calibration. The same applies to the measurement of the output signal from the instrumented striker during the calibration. It may be the case that a

\footnotetext{
${ }^{1}$ Note that resonances in the striker and sample response can lead to oscillation of the force during impact, as shown in Ref [2]; however, the amplitude of force oscillation assigned based on a static calibration will in general not be correct.

${ }^{2}$ This is not to be confused with time-independence. Time-invariance means that the properties of the system are not changing in time.
} 
correction to the dynamic sensitivity of the striker under the conditions of its calibration is required in order to get the sensitivity applicable to a given instrumented test; the uncertainty of any such correction must also be included.

(b) The added uncertainty in performing the instrumented Charpy test must be evaluated and incorporated. This includes for example all significant uncertainty contributions that may arise from electronic noise and environmental influences such as vibration, temperature, atmospheric pressure and electrical interference.

Both uncertainty contributions (a) and (b) must be propagated to the final force values obtained from an instrumented Charpy test, a procedure which may be quite involved [11]. In this Technical Note, scope is limited to contribution (a), that is, to the calibration of the instrumented striker, and furthermore, propagation of this uncertainty is not discussed. We note that it is not necessary that uncertainties from each significant source are individually evaluated, only that the total uncertainty due to them is determined.

It is also important to mention that unlike the static sensitivity, the dynamic sensitivity of the striker may in general be different when it is mounted in the Charpy machine (in-situ) than when it is removed from the machine (ex-situ), and may be different for each machine in which the striker is mounted. This is because the dynamic response to an impact is the response of the entire machine structure. The implication is that the instrumented striker should preferably be dynamically calibrated while mounted in the Charpy machine in which it is to be used. In some cases, differences from one Charpy machine to another may be small.

The remainder of this Technical Note is organized as follows. In Section 2, we discuss established methods for calibrating instrumented Charpy strikers, and in Section 3 we describe a number of potential methods for performing a low-uncertainty SI-traceable dynamic calibration of instrumented strikers. In Section 4, we describe an exercise carried out to test two of these methods using a mock Charpy machine, and report the results of this exercise, as well as the implications for a SI-traceable dynamic calibration of Charpy instrumented strikers. In Section 5, we describe ongoing activities to apply these lessons to the calibration of instrumented Charpy strikers at NIST. In Section 6 we briefly state some preliminary conclusions. 


\section{Established Methods for Calibrating Instrumented Charpy Strikers}

\subsection{Static Calibration}

The most straightforward methodology for calibrating an instrumented Charpy striker consists in disassembling the striker from the Charpy machine, mounting it on a universal testing machine with the aid of suitable grips or fixtures, and statically applying different values of force at the nominal impact location. The striker is loaded against an undeformed Charpy specimen ${ }^{3,4}$, and force is applied in several steps up to the maximum force expected during a test (typically, $30 \mathrm{kN}$ to $35 \mathrm{kN}$ ). This procedure is called static calibration, and is specifically mentioned as the primary option by both ISO 14556:2015 [12] and ASTM E2298-15 [4]. Both standards, however, recommend performing the static calibration with the striker mounted in the pendulum assembly (in-situ calibration).

At NIST, strikers are statically calibrated by applying forces between $0.5 \mathrm{kN}$ and 35 $\mathrm{kN}$. Force values, which are provided by a calibrated load cell, are then correlated to the voltage signals returned by a strain gage bridge on the instrumented striker. These signals are conditioned and amplified by the same measuring chain (signal conditioner/amplifier, digitalto-analog converter) used when performing instrumented Charpy tests. The conversion factor $(\mathrm{kN} / \mathrm{V})$ or calibration function for the striker is obtained by linearly or quadratically fitting the average of three calibration runs as a function of the corresponding values of applied force. An example of calibration curves is shown in Fig. 2 for one of the NIST instrumented strikers. Static calibration treats the striker sensitivity as a unique relationship between force and voltage that is independent of the loading force-time profile. This is in general not expected to be correct.

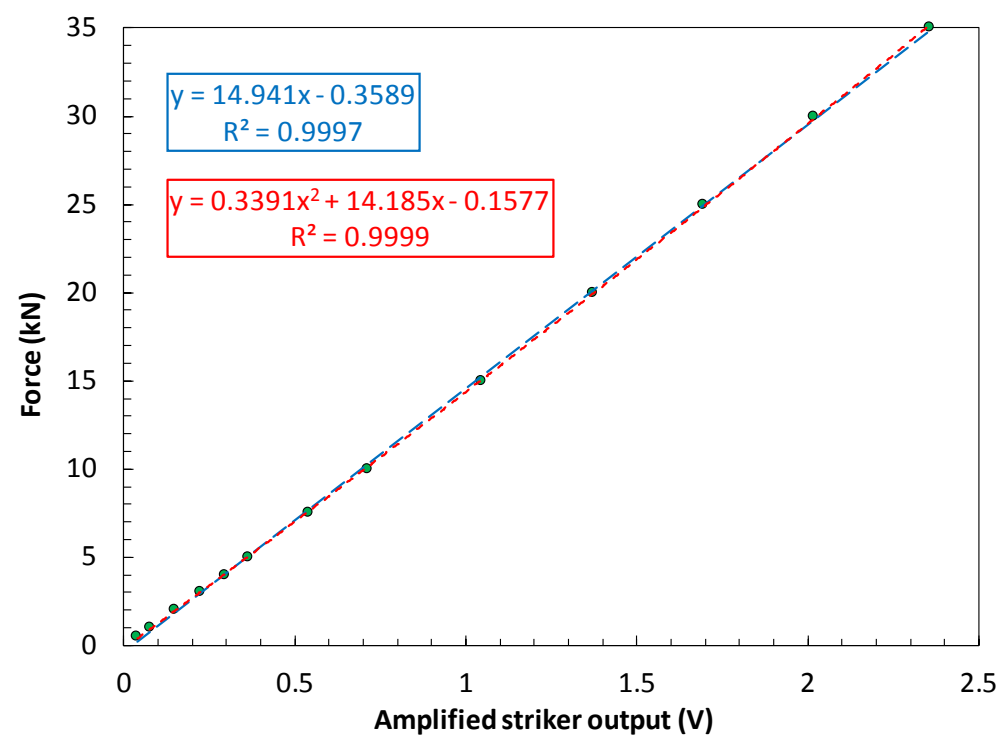

Fig. 2. Example of relationship between applied force and striker output obtained from the static calibration of a NIST instrumented striker. A linear and a quadratic fit are shown in the figure.

\footnotetext{
${ }^{3}$ The Charpy specimen used for the static calibration is generally untested. However, before actual voltage values are recorded, the striker is loaded until its end-of-scale ( $35 \mathrm{kN}$ to $40 \mathrm{kN}$ ) and subsequently unloaded. Therefore, when the process of static calibration is initiated, the specimen is already plastically indented.

${ }^{4}$ Note that ISO 14556:2015 advocates the use of a grooved counter piece opposite the striker during calibration, instead of a flat surface (such as an undeformed Charpy specimen). The radius of the groove is slightly larger than that of the striking edge.
} 


\subsection{Energy Adjustment Method}

This method [3] is based on the fact that the integral of the force applied to the specimen over displacement corresponds to the work done in breaking the specimen, i.e., the energy absorbed during the test. Since the calculation of the Charpy absorbed energy from the difference between fall angle and rise angle of the pendulum is less uncertain than the force measurement, correcting the indicated force values (by applying a uniform correction factor) to obtain an integrated instrumented energy equal to the angle-based absorbed energy can be expected to provide improvement. Since it is difficult to directly measure the displacement of the contact point between striker and specimen during a Charpy test, the displacement (or specimen deflection), $s$, is usually inferred from the force-time data, by using a pendulum effective mass $m$ to convert force to acceleration, and integrating acceleration twice to obtain displacement:

$$
\begin{aligned}
& v(t)=v_{0}-\frac{1}{m} \int_{t_{0}}^{t} F(t) d t, \\
& s(t)=\int_{t_{0}}^{t} v(t) d t,
\end{aligned}
$$

where $v$ is velocity, $v_{0}$ is velocity at impact, and $t_{0}$ is the time of impact.

Significant improvement of instrumented Charpy data has been demonstrated in terms of better agreement between instrumented Charpy tests performed at different institutes (round-robin exercises) when this correction is implemented [3], although discrepancies remain higher than desired. The Energy Adjustment Method based on an inferred displacement has been incorporated in the ASTM E2298-15 standard [4], and is actually required when the discrepancy between angle-based and instrumented absorbed energies is between $15 \%$ and $25 \%$, or between $1 \mathrm{~J}$ and $2 \mathrm{~J}$, whichever is larger. However, the determined sensitivity remains a time- and frequency-independent function, which in general is not expected to be correct.

\subsection{Machine Compliance Method}

The knowledge of the compliance of a Charpy machine allows determining the relationship between striker output and applied force, under conditions which correspond to higher loading rates than the conventional static calibration (see section 2.1).

Like the Energy Adjustment Method described in section 2.2, this method makes use of the absorbed energy measurements of the Charpy machine to calibrate the force indication. The principle of this method, which was originally denominated "dynamic calibration of an instrumented tup" by Ireland [5], consists in matching a calculated peak impulse force, from a purely elastic low-blow test on an unnotched specimen, with that obtained by the instrumented signal [6].

For a rigorously elastic impact test of an unnotched Charpy specimen (see an example in Fig. 3), the maximum force, $F_{\mathrm{m}}$, is calculated from the following relationship, which is strictly valid for elastic energy absorption:

$$
F_{m}=\sqrt{\frac{2 W_{0}}{C_{M}+C_{S}}}
$$


where $W_{0}$ is the maximum available kinetic energy, $C_{M}$ is the machine compliance (measured by several methods proposed by Ireland in [5]), and $C_{s}$ is the specimen compliance, which for an unnotched Charpy-type specimen is given by:

$$
C_{s}=\frac{S^{3}}{48 E I} \quad \text {, }
$$

with: $\quad E=$ Young's modulus (in $\mathrm{MPa}$ );

$S=$ span, or distance between the machine anvils (in $\mathrm{mm}$ ); and

$I=$ area moment of inertia (in $\mathrm{mm}^{4}$ ), expressed as:

$$
I=\frac{B W^{3}}{12} \quad,
$$

with $B, W=$ specimen thickness and width (in $\mathrm{mm}$ ).

Compliance-based conversion factors obtained by this method, reported in [6] for various NIST instrumented strikers, were found to be systematically lower than the corresponding static conversion factors obtained by static calibration. Differences were however in all cases lower than $5 \%$. This is in line with the findings of another investigation [13], which showed that the conversion factor between applied force and striker output (i.e., the striker sensitivity) tends to decrease as the loading rate increases.

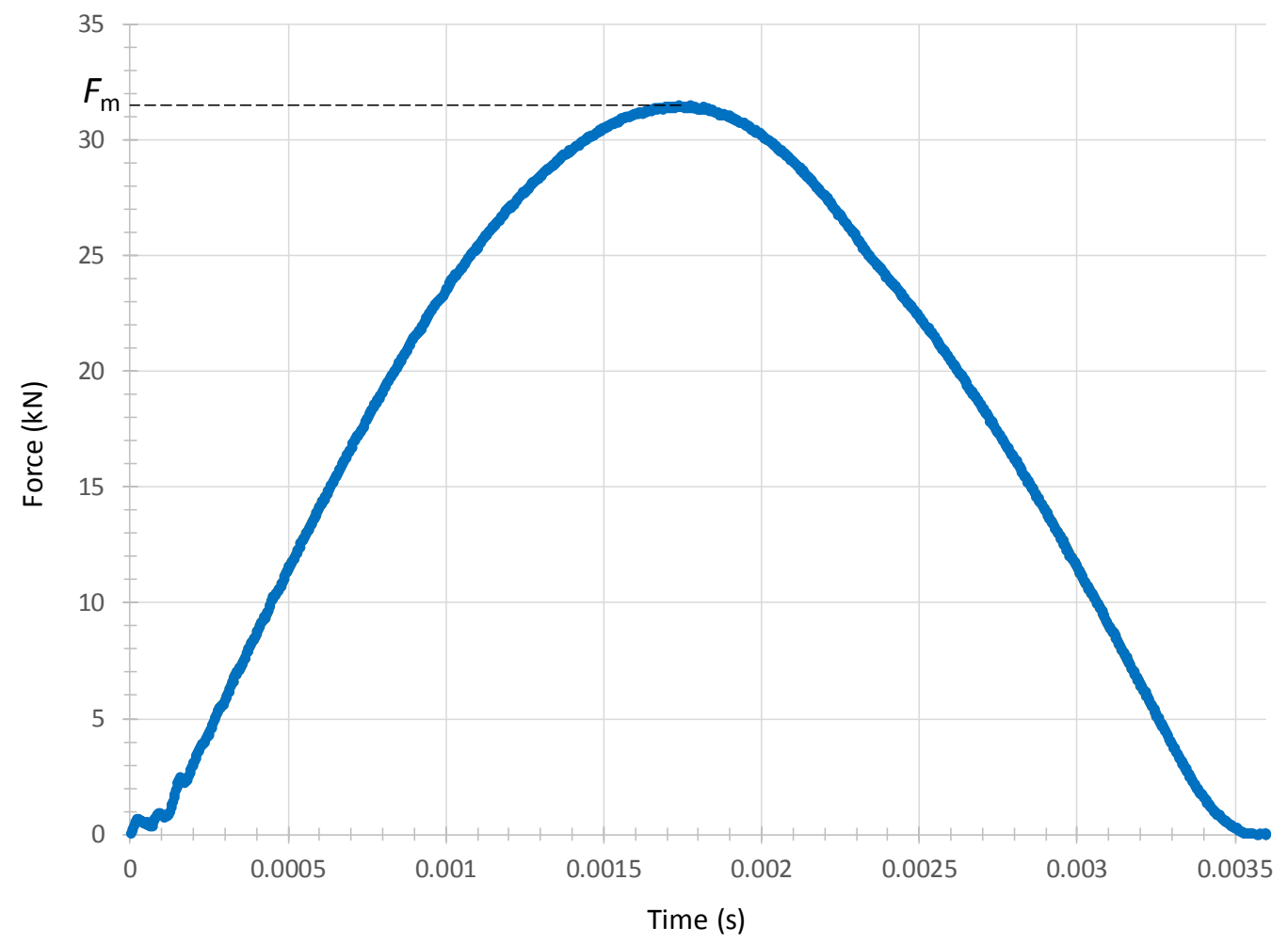

Fig. 3. Example of a force-time curve obtained from an elastic low-blow test performed on an unnotched Charpy specimen for the measurement of the compliance of a Charpy machine.

Like the energy adjustment method, the compliance method replaces the static sensitivity with an "averaged" dynamic sensitivity, which may be expected to provide some 
improvement; however, the determined sensitivity remains a time- and frequencyindependent function, which in general is not expected to be correct. 


\section{Possible Options for an SI-Traceable Dynamic Calibration}

There are a variety of possible methods to obtain an SI-traceable dynamic calibration of an instrumented Charpy striker, and we discuss here a few of these that appear, in the authors' opinion, relatively practical to achieve. Ease of implementation is a critical consideration for the usefulness of a dynamic calibration method. In this vein, while it is not the focus of this Technical Note, we mention that the design of the Charpy striker and the machine play a key role. If a Charpy instrumented striker and machine can be designed such that the sensitivity frequency-response function is frequency-independent (constant amplitude and linear phase) from $0 \mathrm{~Hz}$ up to the highest frequency necessary to capture all features of interest in the instrumented Charpy tests to be conducted, then a static calibration can provide valid lowuncertainty SI-traceable force measurements, obviating the need for a dynamic calibration. If the frequency-response function is frequency-independent down to a low frequency (e.g. a few 10's of Hertz) but not to $0 \mathrm{~Hz}$, then a static calibration is not sufficient but a timeindependent dynamic calibration, using for example the energy adjustment method or a peakimpulse-force calibration, would be sufficient.

\subsection{Machine Compliance Method}

As described in Section 2.2 above, this method is based on requiring that the instrumented absorbed energy be equal to the value calculated on the basis of the fall and rise angles of the Charpy pendulum ${ }^{5}$. Since traceability of the angle-based absorbed energy measurement is relatively straightforward to achieve, this can in principle provide a "weak" traceable force calibration (in the sense that only the integral of the force is traceable, not the force itself). However, this requires a traceable determination of displacement of the contact point between specimen and striker during the impact. As a direct measurement is difficult, a theoretical model is typically used. As previously discussed, the usual approach ${ }^{6}$ is to use the pendulum effective mass $m$, which relates the force $F$ and the acceleration $a$ via Newton's $2^{\text {nd }}$ law, $F=m \cdot a$. For traceability, the absolute uncertainty of this effective mass must be quantified. Also, in propagating the uncertainty, the correlation between force and inferred displacement needs to be taken into account. Implementing a direct measurement of the contact point displacement would overcome these drawbacks.

Another shortcoming of the method, and one that is of a fundamental nature, is its "weakness", due to the fact that only the integral of the force and not the force itself, is traceable. In other words, there are many different ways to manipulate a force-displacement trace to give the desired value of the integral. The simplest method, and the one generally used, is to perform a uniform scaling of the force values. However, this will not correct, for example, incorrect oscillations in the force data due to resonant response of the striker. Whatever the method used to manipulate the force data, quantifying the uncertainty due to the shortcomings of the manipulation method is difficult.

\footnotetext{
${ }^{5}$ This is per se an engineering approximation, since it is known that the two energies (angle-based and instrumented) should not be identical. The former energy contains contributions which are unrelated to the fracture process, such as pendulum vibrations, secondary specimen/hammer impacts, etc. [14]. In principle, these effects could be corrected for, but in practice their magnitudes are difficult to determine.

${ }^{6}$ The ASTM E2298 standard [4] also specifies the alternative of direct measurement of the displacement of the striker with respect to the anvil. For such a measurement, if the displacement is not measured directly at the point of contact, a model is required to relate the displacement at the contact point to the measured displacement.
} 


\subsection{Cut-Wire Method}

One means of applying a known dynamic force to an instrumented striker is to use the breaking of a wire under tension that is attached to the striker, as illustrated in Fig. 4. The force before cutting the wire is static and known, and the force after the cut is zero, apart from the contribution of the inertia of any wire that remains connected to the striker after impact. Use of a thin wire and a large enough static force ensures that the post-cut force due to the inertia of the portion of wire that remains attached is negligible ${ }^{7}$.

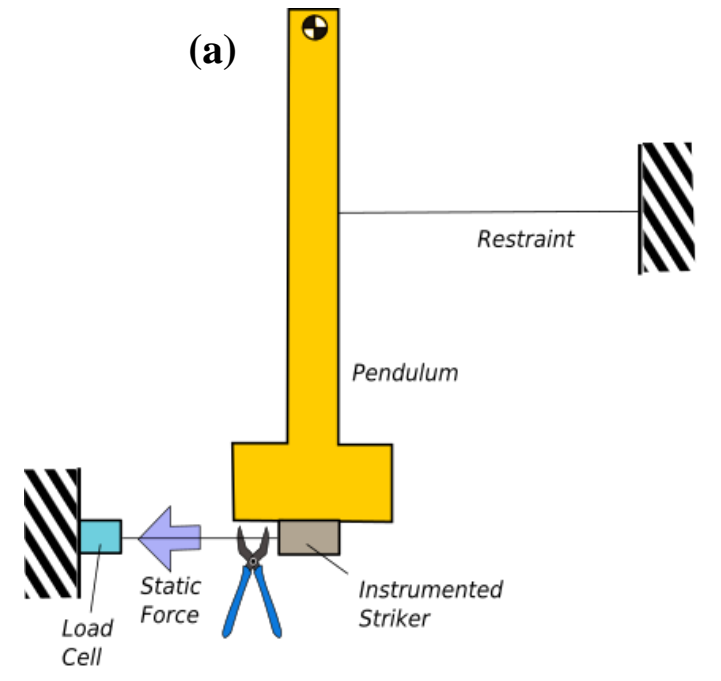

(c)

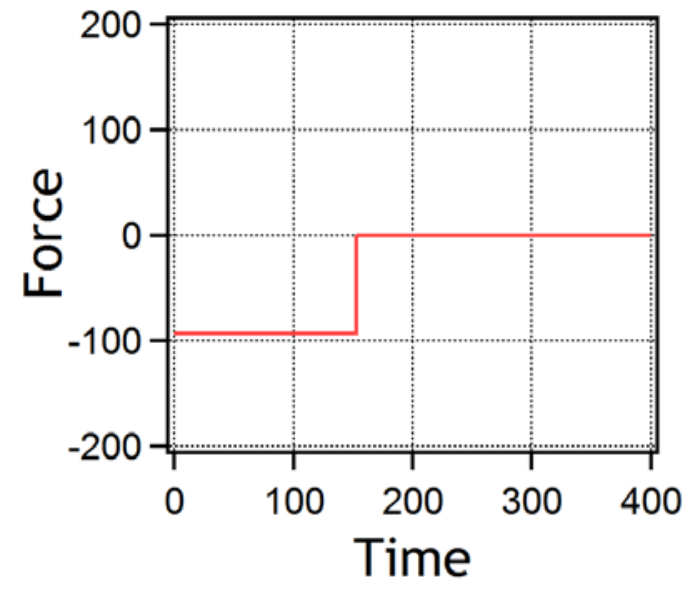

(b)

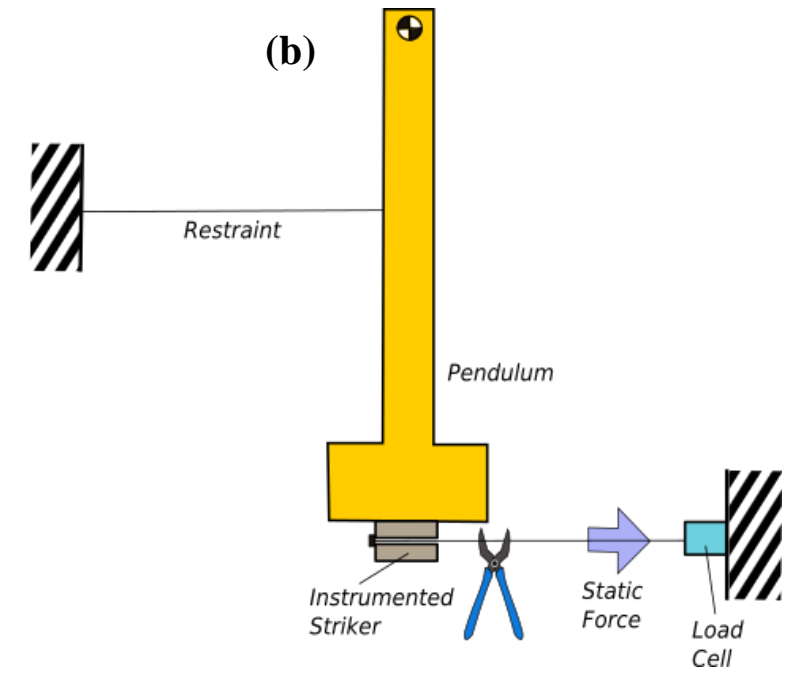

(d)

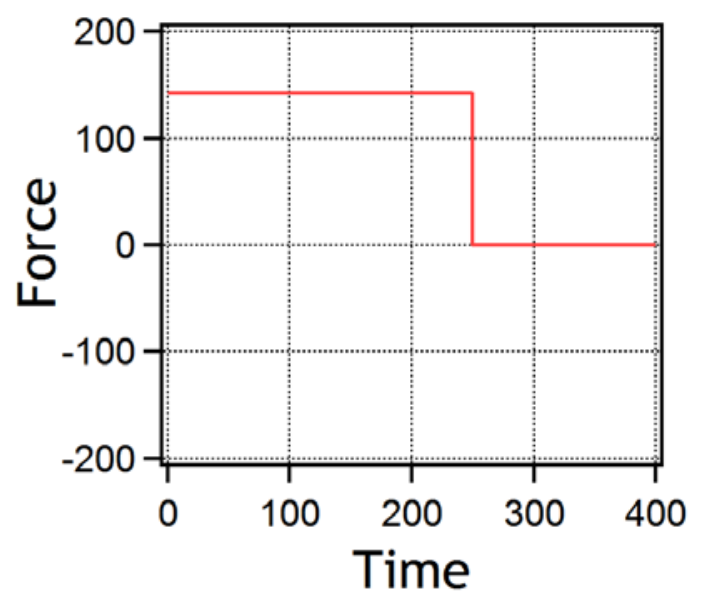

Fig. 4. Cut-wire method with (a) tensile loading and (b) compressive loading; (c) and (d) show idealized generated force steps by configurations (a) and (b) respectively, in arbitrary units.

Thus, the magnitude of the applied force step is known. While the detailed force evolution during the rapid step is unknown, for a sufficiently fast step, the step can be approximated as instantaneous. More precisely, the frequency spectrum of the pulse corresponding to the derivative of the applied force step will be known to be frequency-independent with high

\footnotetext{
${ }^{7}$ If there are additional force transmission components that remain connected to the striker after the fracture, the mass of these may result in a non-negligible post-cut force.
} 
accuracy, up to some frequency limit $f_{l}$ set by the pulse duration, $f_{l} \propto 1 / \tau$, where $\tau$ is the pulse duration. ${ }^{8}$ To the extent that the pulse duration is not exactly known but only bounded, this possible deviation is an uncertainty contribution to the transfer function of the striker determined by this method. To obtain a single step of minimum duration, it is necessary that the wire be single-strand. Cut wires have been used to generate force steps with durations of tens of microseconds [15, 16], and the method has been used for 2-percent level calibration of a force transducer up to a frequency of $5 \mathrm{kHz}$ [16].

There are a number of challenges in successfully applying this method. One is the attachment of the wire to the instrumented striker, which typically requires a hole to be drilled into the striker. While this is not difficult, the challenge is locating the attachment point as close to the impacting surface of the striker as possible. Any difference in the location of the attachment and the impact surface will lead to differences in the dynamic response of the striker during impacts with respect to the response during calibration, and hence an increase in the calibration uncertainty, particularly in the high-frequency range. Similarly, a difference in the applied force profile during test and calibration can cause a difference in the striker sensitivity under the two conditions, as was previously observed (see e.g. [17]); the presence of an attachment hole may exacerbate this. Another consideration is that if the wire applies a tensile load to the striker as shown in Fig. 4(a), the requirements on linearity of the striker response are increased, as the force on the striker during a Charpy impact test is compressive. Additionally, in order to calibrate the pendulum at the bottom of its swing, the pendulum must be restrained during application of the static load. The attachment point of the restraint should be located sufficiently far from the loading point, so that the transit time of elastic waves between the two points is long enough and doesn't affect the lowest frequency required from the dynamic calibration. Lastly, the time and effort of repeatedly securing and cutting wires makes it tedious to perform many repeated calibrations, which may be required in order to provide the desired signal-to-noise ratio. All these factors will contribute to the uncertainty obtained in calibration by this method.

\subsection{Mass Impact Method}

Another simple principle by which a known dynamic force could be applied to a Charpy striker is to arrange an impact between the striker and an unrestrained known mass $m$, measure the acceleration $a(t)$ of the mass during the impact, and use Newton's second law, $F(t)=m \cdot a(t)$, to deduce the force $F(t)$. Two common means that can be used for measuring the mass acceleration during impact are by attaching an accelerometer, and by using a laser interferometer. Generally, the acceleration must be measured at one or at a few locations on the mass, and corrections are required for the effects of any rigid body rotation and for the nonuniformity of acceleration throughout the mass at any instant; the latter effect increases in significance with mass size (for given material properties) and with the frequency range of interest. The uncertainty of this correction contributes to the overall calibration uncertainty. The accuracy of an accelerometer measurement is limited by the calibration uncertainty of the accelerometer as well as an imperfect coupling of the accelerometer to the mass; the latter being of increasing concern with increasing frequency, see e.g. [18]. The uncertainty of the (single-point) acceleration measurement with an accelerometer is thus typically in the range

\footnotetext{
${ }^{8}$ In detail, the values of $f_{l}$ and the proportionality constant depend on the specified threshold of deviation from frequency-independence and on what constraints can be put on the pulse shape.
} 
of a few percent. An interferometer can provide lower uncertainty, but is more complex and expensive.

It is generally easier to measure the acceleration during impact if the mass is guided. Additionally, in order to impact the Charpy striker as mounted in the Charpy machine and in the same location and direction as when the striker impacts a sample, the mass must be traveling horizontally at impact. Two means of achieving guided horizontal impacts are a bearing guide (in the form of a rail or tube) and a pendulum suspension. In either case, the parasitic force due to air drag must be considered if the impact velocity is sufficiently high. In order to achieve short duration impacts, small masses and high velocities are desired [2], while still avoiding plastic deformation. Additional parasitic forces that will contribute to the force uncertainty may come from cables connected to the mass in the case where an accelerometer is used, and bearing friction (an air or magnetic bearing is recommended in order to minimize this).

\subsection{Dynamically Calibrated Transfer Transducer}

Another approach [8] is to use a dynamically calibrated force transducer, i.e. a transfer transducer, to apply a dynamic force to the Charpy striker, thereby transferring the calibration to the striker. While this method is simple in principle, SI-traceable broadband dynamic calibration of force transducers has only recently been demonstrated [16, 19-20], and is not routinely available. Methods that could be used for calibrating the transfer transducer include those described in Sections 3.2 and 3.3 above. As noted above, it is important that the instrumented Charpy striker be calibrated in the configuration in which it will be used, i.e. mounted in the Charpy machine. Similarly, it is important that the dynamic calibration of the transfer transducer be applicable to the manner (boundary conditions) in which it will be used. This is most easily achieved by calibrating and using the transfer transducer $^{9}$, so that it is only weakly coupled (i.e. by a flexible coupling) to the external structure; in this case, the dependence of the transducer response on the supporting structure is limited to low frequencies and the response in the frequency range of interest is not affected. For example, the transfer force transducer may be suspended by wires when calibrated and used, so that its (pendulum mode) natural frequency is on the order of $1 \mathrm{~Hz}$ or less. Similarly, the flexible handle of an impact hammer, discussed further below, assists in decoupling it from its environment.

The dynamic force by which the transfer is done could have a wide variety of forms, for example stepped-sine, chirp, or impulse; the simplest to apply is probably an impulse achieved by impacting the two transducers together. As the dynamic force-time trace used for the transfer will generally not be the same as that in the Charpy test when the striker is used, the calibration is nevertheless in the form of an impulse response function (time domain) or a complex frequency response function (frequency domain). In transferring the calibration from the transfer transducer to the Charpy striker, the actual force applied does not need to be calculated. The ratio of (frequency domain) output signals of the two instruments transfers the calibration from the transfer transducer to the Charpy striker regardless of the exact force value [16], as long as both instruments are linear and time-invariant.

\footnotetext{
9 “Transducer" is interpreted here to include all components that remain coupled to the sensing element in both calibration and use, e.g. a reaction mass to which the transducer remains connected.
} 


\subsection{Closing Comments}

As noted in the introduction, determining the dynamic sensitivity (impulse response function or frequency response function) of a Charpy instrumented striker, does not immediately provide the force-time trace returned from a voltage-time trace obtained from the instrumented striker. This is because the deconvolution must be performed, and this deconvolution is often unstable with respect to noise in the data [9]. Regularization, essentially a nonlinear filtering process, of the deconvolution is generally required. A further challenge is to determine the uncertainty on the determined force data given an uncertainty of the calibration and an uncertainty of the striker output voltage measurement; such propagation of uncertainties through a regularized deconvolution is currently a subject of active research (see e.g. [11], [21]). 


\section{Dynamic Calibration Exercise}

In order to develop a methodology for performing the dynamic calibration, two of the above methods, the use of the cut-wire calibration method (Section 3.2) and a transfer force transducer (Section 3.4), were tested on an old instrumented Charpy striker installed on a simple mock Charpy machine constructed for this study, a rendering of which is shown in Fig. 5.

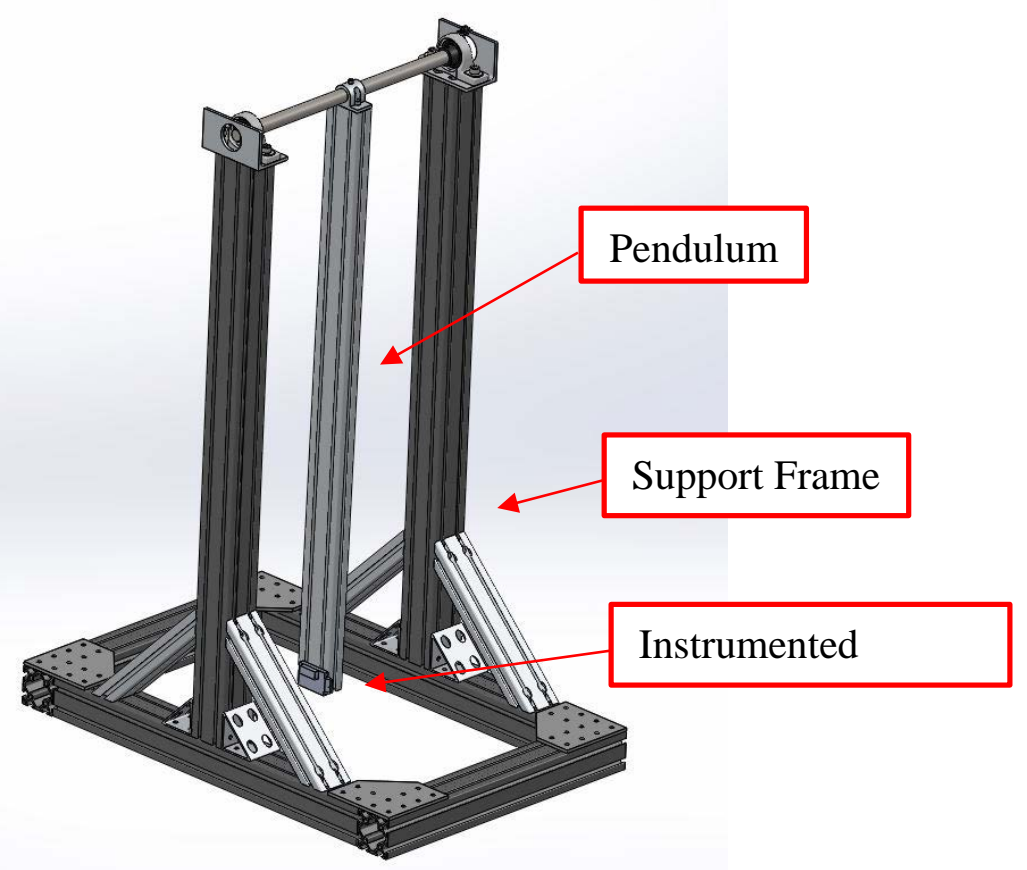

Fig. 5. Computer rendering of mock Charpy machine used for testing calibration methodologies.

\subsection{Cut-Wire Calibration Test}

The apparatus used to perform the cut wire calibration tests is shown in Fig. 6. A frame was constructed around the mock Charpy machine, on which a calibrated load cell mounted on an $\mathrm{XY}$-adjustment stage was supported. A coupling with a small hole in it was used to connect a nylon wire to the load cell. A small transverse hole was drilled into the striker near the impact surface, and centered in the impact area, as shown in Fig. 6. Approximately halfway up the pendulum arm from the striker, an attachment point was provided for a restraining cable. This restraining cable was tensioned by a winch; turning the winch applied a static tensile force to the Charpy striker, which was measured by the load cell. Since the nylon cable was elongated by the applied force, axial adjustment of the load cell position using the adjustment stage on which it was mounted was required in order to keep the striker at its bottom position (i.e. the position in which it impacts a specimen). In this manner, a predetermined force was applied to the striker, and then the nylon cable was severed, causing a rapid step from the applied static force to zero force.

Two different nylon wire configurations were used: 
a) Single-strand round nylon wire of diameter $0.72 \mathrm{~mm} \pm 0.02 \mathrm{~mm}$ (rated for $220 \mathrm{~N}$ of force) was passed through the hole in the striker and knotted at one end, and at the other end passed through a hole in an adapter connected to the load cell and knotted. The single strand was cut when the applied tensile force was $90 \mathrm{~N} \pm 3 \mathrm{~N}$ (although in two cases the wire broke at a lower force value).

b) Multi-strand cable of the proprietary commercial material Spectra ${ }^{10}$, of approximately elliptical cross section with minor and major dimensions of $0.52 \mathrm{~mm} \pm 0.05 \mathrm{~mm}$ and 0.87 $\mathrm{mm} \pm 0.05 \mathrm{~mm}$ (rated for $900 \mathrm{~N}$ of force), was passed through the hole in the striker and formed into a loop of a few inches in length. Single-strand, approximately round, nylon wire of diameter $1.28 \mathrm{~mm} \pm 0.08 \mathrm{~mm}$ (rated for $580 \mathrm{~N}$ of force) was tied at one end to this short loop and at the other end to the load cell adapter. It was found that this configuration provided a higher force capability than just using the single-strand nylon wire, likely due to the effect on the nylon of imperfectly smoothed edges of the hole in the striker. The single-strand nylon wire was cut when the applied tensile force was $160 \mathrm{~N} \pm 3 \mathrm{~N}$.

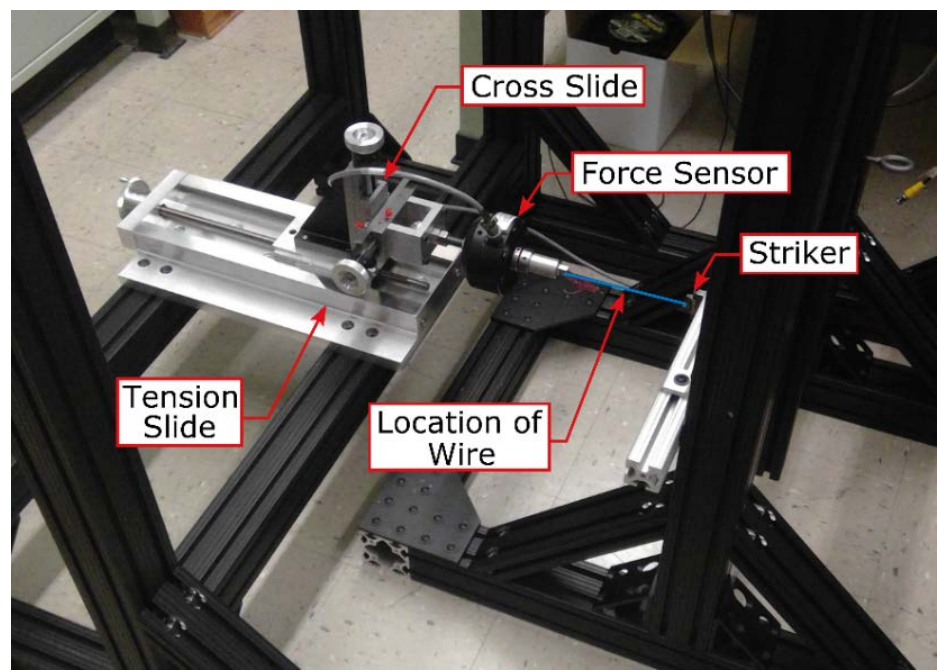

Fig. 6. Photograph rendering of the setup used for testing the cut-wire calibration method.

The data acquisition system used for this test is shown in Fig. 6. The voltage output of the calibrated load cell was measured using a 6.5-digit DC multimeter operating at a 10 sample-per-second measurement rate. The voltage output from the instrumented striker bridge amplifier was measured by a fast-sampling DC multimeter operating at a measurement rate of 50000 samples per second. No anti-aliasing filter before the voltage digitization was used in this cut-wire exercise; as the passband of the amplifier output extended to well above $25 \mathrm{kHz}$, this could have increased the noise level in the measurement bandwidth. The acquisition of the striker output voltage was triggered by an electrical contact on the cutters used to sever the nylon line. Voltage traces from a typical test are shown in Fig. 8.

\footnotetext{
${ }^{10}$ Certain commercial products are identified in this article in order to describe the experimental procedure adequately. Such identification is not intended to imply recommendation or endorsement by the National Institute of Standards and Technology, nor is it intended to imply that the products identified are necessarily the best available for the purpose.
} 


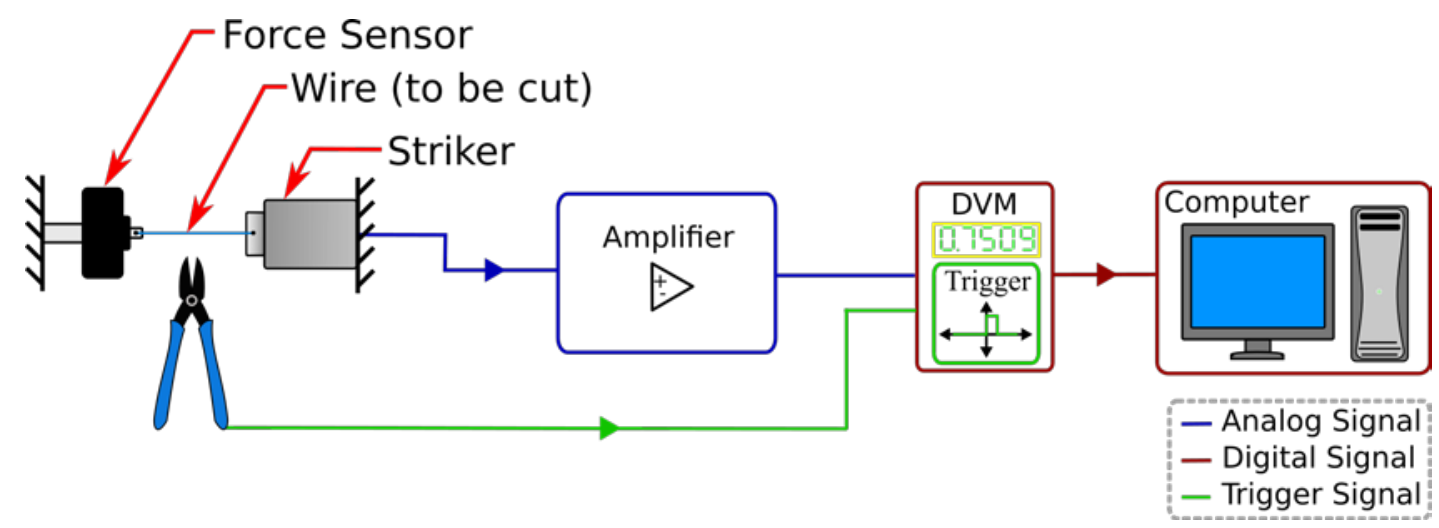

Fig. 7. Data acquisition arrangement used for testing cut-wire calibration method.

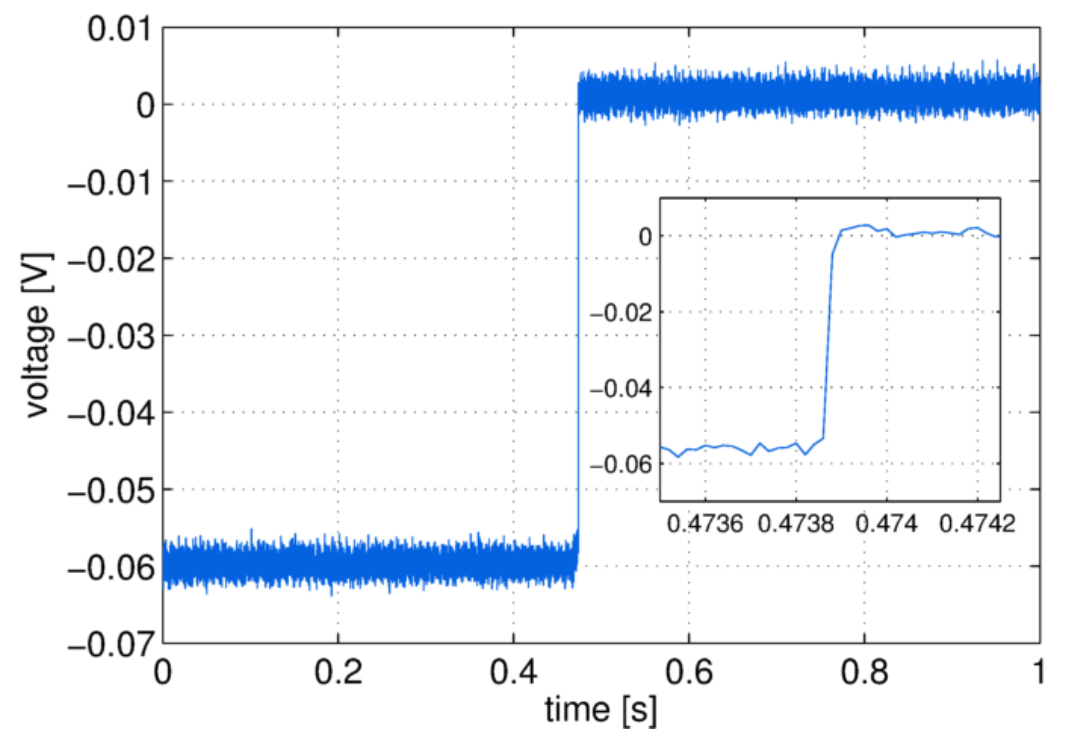

Fig. 8. Voltage trace for an experimental cut-wire calibration test.

The calibration results are shown in Fig. 9. Reasonable agreement between the two cut-wire tests is observed up to $\approx 3 \mathrm{kHz}$, and the observed low-frequency sensitivity agrees with the measured static sensitivity for this striker of $0.70 \mathrm{mV} / \mathrm{N} \pm 0.06 \mathrm{mV} / \mathrm{N}$. The deviation of the two cut-wire configurations from each other is believed to be due to variations in the finite duration of the force steps that are received at the instrumented striker; previous use [16] of single-strand nylon wire to do step calibrations of a force transducer found deviations between different-diameter wires and tensions starting at $\approx 5 \mathrm{kHz}$. The finite step duration could also cause or contribute to the observed rolloff in the sensitivity starting at $\approx 5 \mathrm{kHz}$, as the true excitation force amplitude will decrease with frequency, whereas a frequencyindependent amplitude, corresponding to an instantaneous step, is assumed. 


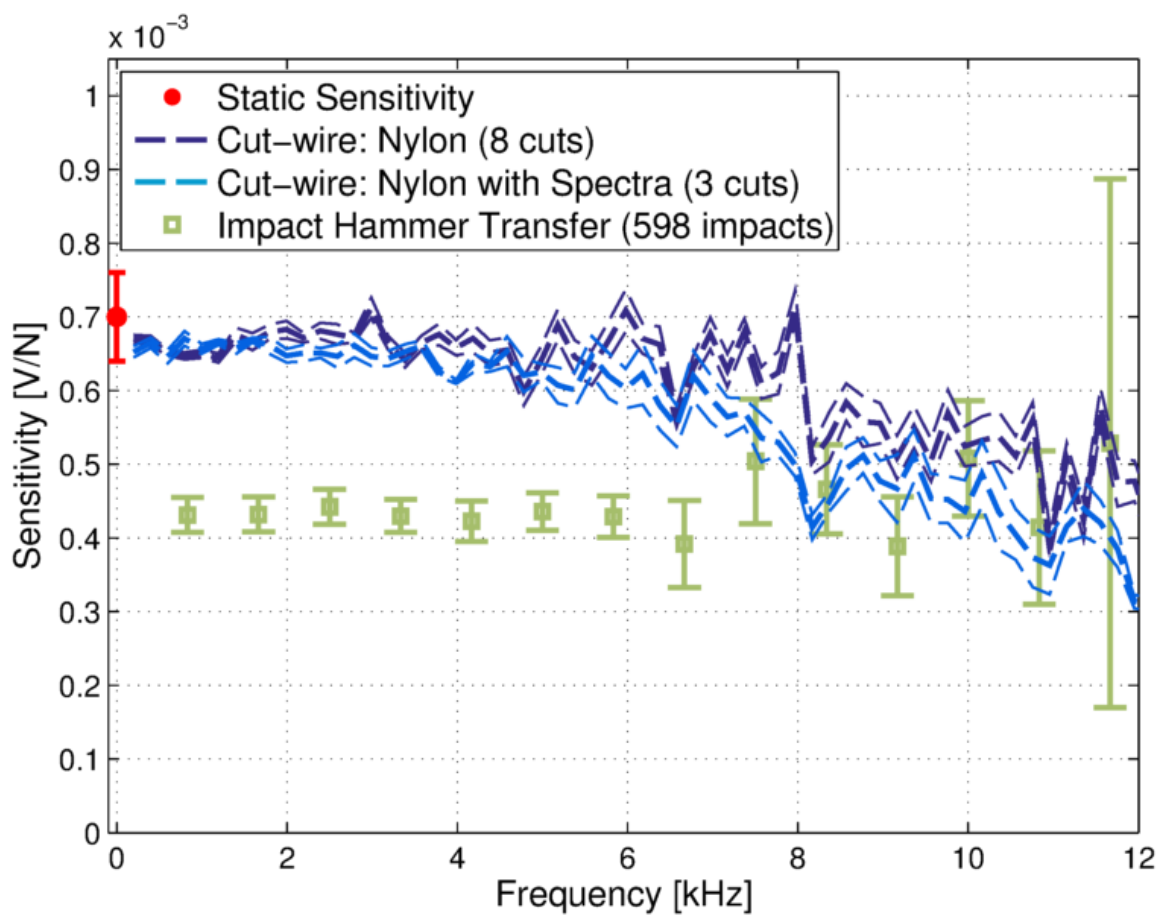

Fig. 9. Experimental calibration spectra for the striker mounted in the mock-Charpy machine. Results are shown for cut-wire and transfer-standard based calibration tests. The error bounds, shown as thin dashed lines for the cut-wire traces and as vertical bars for the impact data, are the scatter (1 standard deviation) of the acquired data.

\subsection{Calibrated Transfer Transducer Test}

For this method, a calibrated piezoelectric impact hammer (or impulse hammer) was used as the transfer transducer. Such a hammer is a force transducer mounted on a reaction mass, supported by a flexible handle, and often incorporating an accelerometer and integrated electronics to perform partial cancellation of inertial forces due to acceleration of hammer parts. A drawing of such a transducer is shown in Fig. 10. The impact hammer used in these tests had an operating force range of $2200 \mathrm{~N}$. Using an aluminum impact cap of $6.3 \mathrm{~g}+/-0.1$ $\mathrm{g}$, we generated pulses on the maraging steel instrumented striker of duration ( $1 \%$ peak force width) between $210 \mu$ s and $260 \mu$ s, containing significant force spectral content $(-40 \mathrm{~dB}$, i.e. $1 \%$ of the low-frequency value) up to approximately $13 \mathrm{kHz}$. The hammer had been calibrated using the cut-wire method as described in [16], with a $k=2$ uncertainty on the sensitivity of $1.4 \%$ at $3 \mathrm{kHz}, 2.1 \%$ at $5 \mathrm{kHz}$, and $5.6 \%$ at $10 \mathrm{kHz}$.

The hammer output voltage and the instrumented striker output voltage were digitized simultaneously at a rate of 500 kilosamples per second, in the arrangement shown in Fig. 11. The digitizer channel used for recording the impact hammer output voltage was the same as in the calibration of the impact hammer; therefore, overall calibration of the digitizer was not necessary. Only noise, any channel-to-channel gain difference, crosstalk, drifts and nonlinearity affect the transfer calibration. 


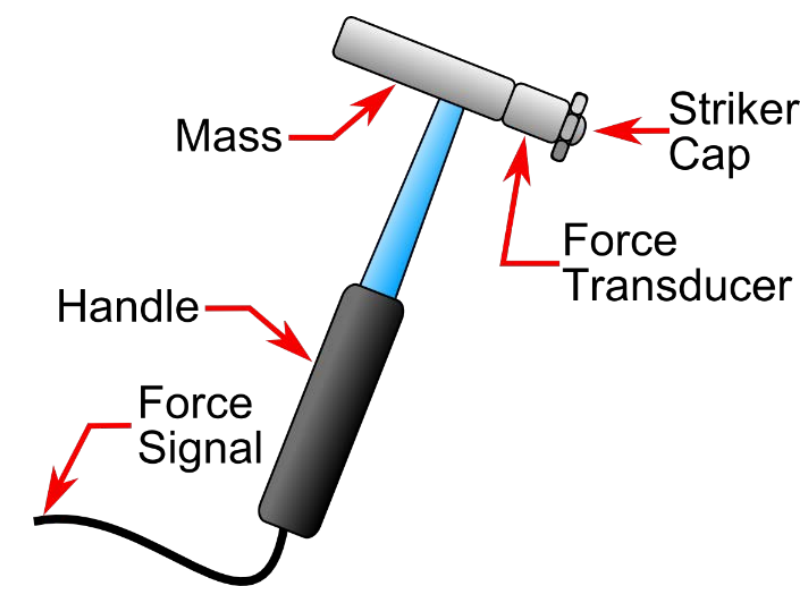

Fig. 10. Typical construction of an impact hammer.

The hammer output voltage and the instrumented striker output voltage were digitized simultaneously at a rate of 500 kilosamples per second, in the arrangement shown in Fig. 11. The digitizer channel used for recording the impact hammer output voltage was the same as in the calibration of the impact hammer; therefore, overall calibration of the digitizer was not necessary. Only noise, any channel-to-channel gain difference, crosstalk, drifts and nonlinearity affect the transfer calibration.

Three different persons (each one of the authors) applied between 100 and 400 strikes of the impact hammer on the device, which were averaged after rejecting under-range, overrange and multiple-impact strikes. The results for each individual, plotted in terms of the ratio of the striker output voltage to the hammer output voltage, are shown in Fig. 12. While the vertical axis units on this plot are not physically meaningful, the important point is that a systematic difference between individuals was observed. This is believed to be due to a high sensitivity of the Charpy striker output signal to the precise location and direction of the impact, coupled with some systematic variation of this among individuals. The sensitivity of the striker output signal to impact location was emphasized by striking it at some locations a few centimeters from the nominal center of the striking region, and observing a change in the sign of the output voltage.

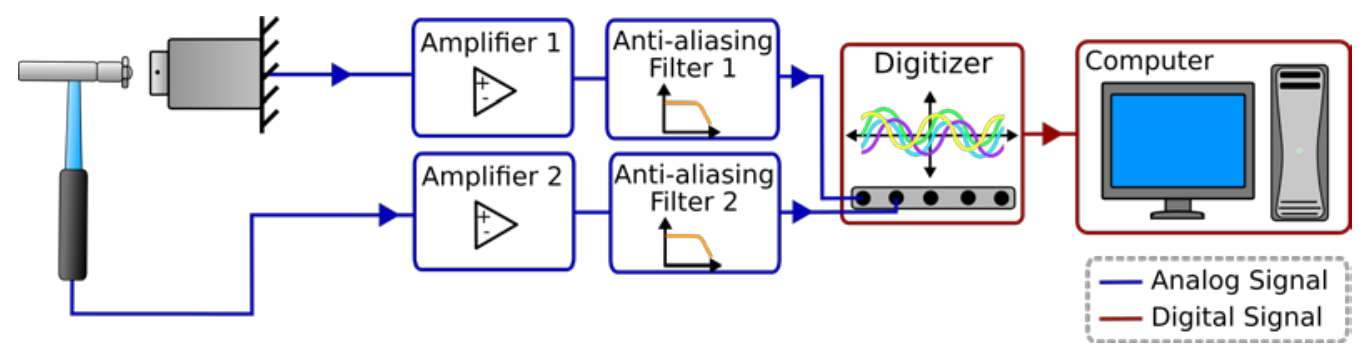

Fig. 11. Data acquisition arrangement used for testing the calibrated transfer transducer method. 


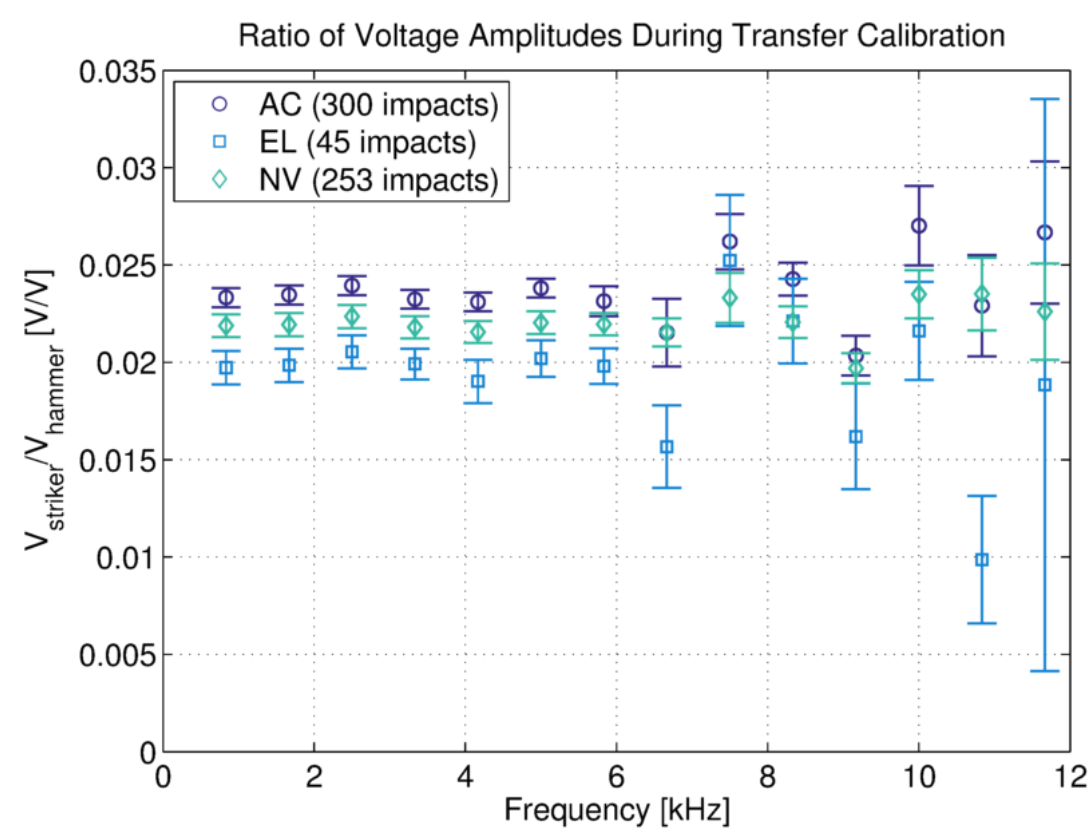

Fig. 12. Dependence of calibration on person swinging hammer, for striker mounted in mock-Charpy machine. Error bars represent 1 standard deviation.

The results of the test calibration using the transfer transducer (impacts from all 3 individuals averaged together) are shown in Fig. 9. These results, which differ from the results of the cut-wire test by about $50 \%$ in the low-frequency range, are considered as erroneous, with the error believed to arise from the sensitivity of the striker response to precise impact angle and location. Since the cut-wire force is applied to the Charpy striker at or close to its most sensitive point, the averaging of the impact hammer results over position and angle results in a lower indicated sensitivity.

The low signal-to-noise ratio (SNR) of the impacts for frequencies above $10 \mathrm{kHz}$ results in large uncertainty of the impact-determined sensitivity in this region. The SNR limit to the sensitivity was primarily set by the striker output signal, for which SNR $>1$ was available up to $\approx 12 \mathrm{kHz}$. The impact hammer output had SNR $>1$ up to frequencies greater than $25 \mathrm{kHz}$. We note that even for the noise level observed, the SNR-limited bandwidth of the striker output signal in an instrumented Charpy test can be higher than the $12 \mathrm{kHz}$ obtained in this exercise, as there may be larger amplitude high-frequency components in the force during an instrumented test (i.e. the signal level could be higher while the noise level remains unchanged).

Even with the large standard deviation of the acquired impact hammer data, there is an apparent trend with increasing frequency in the opposite direction from the cut-wire results. Like the cut-wire results, this could be due to the finite bandwidth of the cut-wire technique, which was the approach used to calibrate the impact hammer transfer standard. This finite bandwidth results in an erroneously-low assigned sensitivity of the transfer standard at high frequencies, and then an erroneously-high assigned sensitivity of the striker when the transfer calibration is performed. 


\subsection{Summary and Lessons Learned}

A number of preliminary conclusions were drawn from this exercise:

(a) The low-uncertainty bandwidth achievable with the cut-wire method is at the present time limited to approximately $5 \mathrm{kHz}$. Some development (e.g. faster severing of the wire, investigation of force step dispersion, attachment of wires made of stiffer material), will be required to increase the bandwidth of this method, which is required for many instrumented Charpy tests. Additionally, reducing the uncertainty even in the frequency range up to $5 \mathrm{kHz}$ will require averaging many cuts, taking hours or days at the achieved rate of approximately 10 cuts per hour.

(b) The extreme sensitivity of the striker response to precise impact location and angle makes calibration with a hand-swung impact hammer challenging. Two steps that can address this are the mechanization of the impact hammer swing to increase the accuracy of the achieved impact location and angle, and redesign of the Charpy instrumented striker to make it less sensitive to impact location and angle.

(c) It is believed that the low-uncertainty bandwidth of the calibration using the impact hammer would also be approximately $5 \mathrm{kHz}$, after the impact location- and angle-sensitivity problems are solved. Increasing this frequency requires calibration of the impact hammer to a higher frequency. This requires some development, although it is believed that this is less challenging than increasing the cut-wire calibration bandwidth; a mass-impact approach is proposed for calibrating the impact hammer to higher frequency.

(d) The impact hammer approach has a large practical advantage in speed over the cut-wire method, allowing hundreds of impacts, with similar spectral force density in an impact as in a cut-wire test, to be collected and averaged in approximately 10 minutes. Automation of the impact hammer could increase this rate to thousands of impacts in approximately 10 minutes.

As a result, it was decided to focus on the transfer transducer method for calibration of Charpy strikers in actual Charpy machines. To achieve this goal, we will develop a massimpact based calibration of the impact hammer transfer transducer, design a new Charpy instrumented striker that is less sensitive to impact location and angle, and construct a mechanical apparatus for swinging the impact hammer.

An additional issue applicable to the calibration methods tested is linearity. Nonlinearity is a general limitation on the validity of a frequency response function or an impulse response function representing the dynamic sensitivity of the Charpy striker, and is of heightened concern if the calibration is to be done in a small part of the operating force range as was the case for the two calibration methods tested. Forces up to $5 \mathrm{kN}$ are generated by the impact hammer transfer transducers used in this study, and forces of approximately $500 \mathrm{~N}$ should be possible using e.g. $0.5 \mathrm{~mm}$ diameter tungsten wire. In contrast, Charpy striker force amplitudes up to $35 \mathrm{kN}$ occur in tests. Larger force amplitudes can be generated by larger impact hammers, at the expense of applied force bandwidth. Linearity of the striker response is thus an additional important design criterion, to aid dynamic calibration. For cases where there is significant nonlinearity, the question of the uncertainty introduced by this nonlinearity becomes important. For the transfer standard calibration method, linearity of the used transfer standard is also relevant. For piezoelectric impact hammers, the 
quasistatic nonlinearity, defined as the deviation of measured voltage values in a quasistatic calibration from a straight-line fit through the calibration data, is often specified as being below $1 \%$ of the full-scale output [22].

The lessons learned in the activity conducted, summarized above, constitute a comparative assessment of the two methods tested, which were initially selected as the most promising to implement within our existing capabilities. Implementation of an alternate method is possible, for example primary calibration based on a mass impact (section 3.3), and has not been assessed within the scope of this work. 


\section{Current Efforts for Dynamically Calibrating an Instrumented Charpy Striker}

\subsection{Design Modifications to Improve Instrumented Striker Performance}

As pointed out in Section 4.3, the striker on which calibration methods were tested has the undesirable trait that its sensitivity is strongly dependent on the precise location where the force is applied to the striker and to force components orthogonal to the design impact axis, which can lead to errors in making static and dynamic measurements. In our experience the sensitivity dependence on impact location is common to many designs of instrumented strikers. The precise location of the applied force during the static and dynamic calibration may not be at the same location as when the striker is used to break samples; thus, the sensitivity measured during the calibration may not be valid while breaking samples. Moreover, when breaking samples, each specimen may not make contact on the same location of the striker due to deformation of the specimen and striker [23]. This sensitivity dependence on impact force-location can lead to significant errors in both static and dynamic response.

We have designed an instrumented striker that has a sensitivity that is relatively constant with respect to variations in the location of the impact forces [24], which reduces both static and dynamic errors. The invariance of the sensitivity to impact location is achieved through the spatial configuration of the strain gages and the corresponding electrical circuit. A photograph of the redesigned striker is shown in Fig. 13 (a), while one side of the strain gage mechanical configuration is shown in part (b). Further details of this device are provided in [24].

A plot of the normalized static sensitivity of the device described in this section compared with that of the device tested as described in Section 4, as a function of impactforce location, is shown in Fig. 14. For both devices, the force was applied $3 \mathrm{~mm}$ below the nominal impact location and $2 \mathrm{~mm}$ above the nominal impact location. The typical-design striker, presented in Section 4, has a sensitivity that deviates as much as $35 \%$ from the nominal value, while the new design deviates by less than $2.5 \%$.

(a)

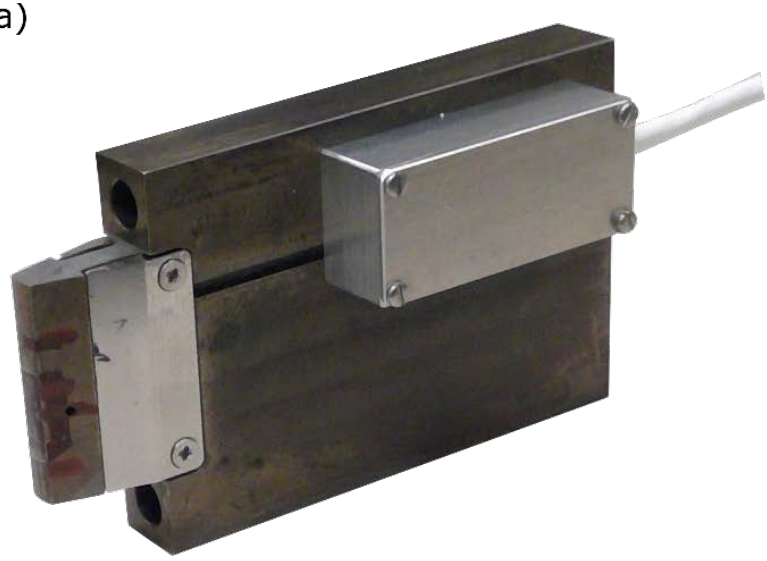

(b)

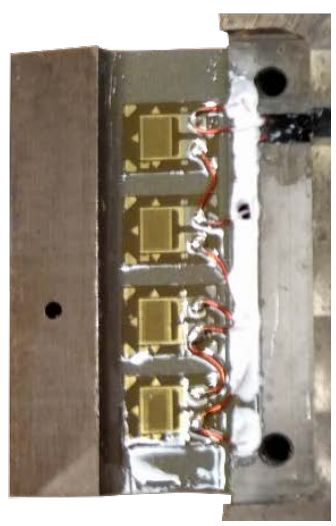

Fig. 13. Photographs of the (a) instrumented striker that was designed to have an invariant sensitivity to impact location and (b) the strain gage configuration on one side of the instrumented striker. 


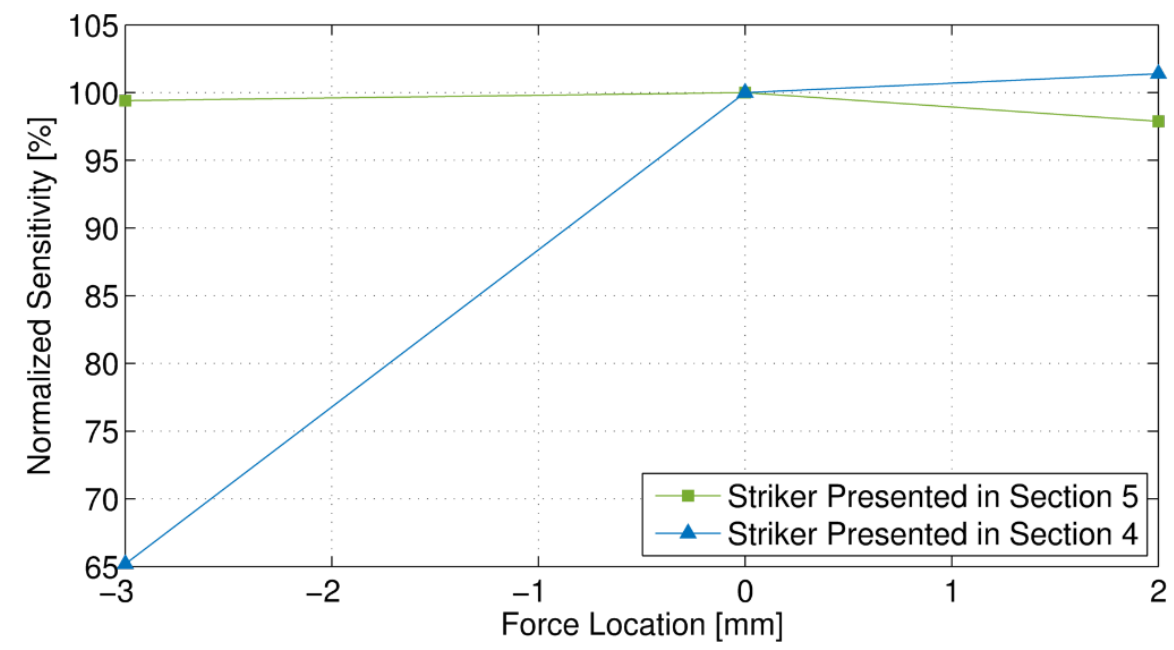

Fig. 14. Comparison of the normalized sensitivity as a function of impact location for the instrumented striker presented in Section 5 to the one shown in Section 4.

\subsection{Extension of Transfer Transducer Calibration Bandwidth and Mechanization of Impacts}

As stated previously, the transfer standard used to calibrate the instrumented striker as described in Section 4.2 was traceably calibrated using a cut-wire approach with a $k=2$ uncertainty $\leq 2.1 \%$ up to $5 \mathrm{kHz}$. In turn, the instrumented striker can only be calibrated up to $5 \mathrm{kHz}$ with the same level of uncertainty or higher, using the transfer standard. Currently, a mechanized impactor system, such as the one shown in Fig. 15, is being developed to further increase the low-uncertainty calibration bandwidth. Initial estimates suggest that the transfer standard can be calibrated up to $20 \mathrm{kHz}$ with acceptable uncertainty using a mechanical apparatus such as this one. The calibration apparatus works by securing the transfer standard (impact hammer) in a pivoting arm, whereupon the arm swings down and strikes a mass that is held in an air bearing. As the mass moves away from the impact hammer, its displacement is traceably measured using a laser interferometer. From this displacement, the acceleration is calculated. Using the acceleration, the mass value, and the voltage recorded from the impact hammer, the sensitivity of the device is then determined.

The mechanized support used in the calibration of the impact hammer also provides a means to control the trajectory of the instrument and the impact location. This reduces both uncertainty and variation in impact angle and location in comparison to when the device is hand-held. The transfer standard can be left in the same apparatus, and the entire assembly can then be transported to the location of the Charpy machine to calibrate the instrumented striker in-situ. 


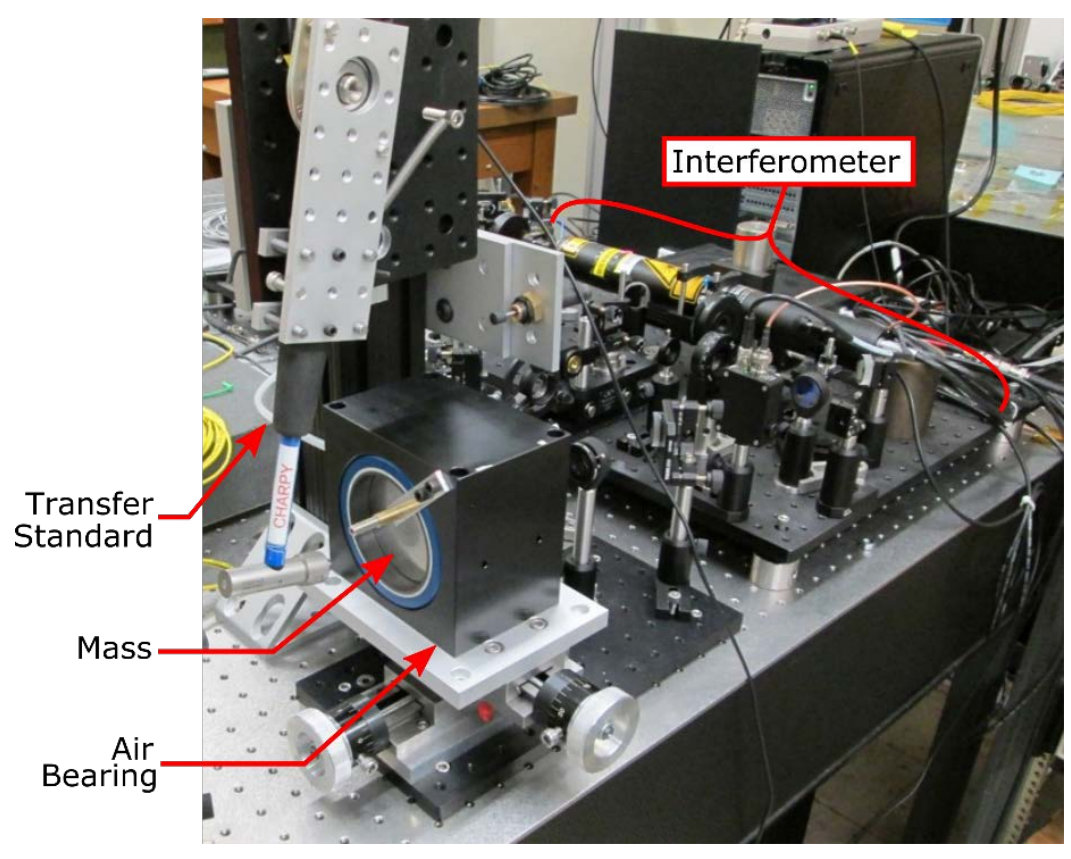

Fig. 15. Prototype mechanical device used to secure the transfer standard, which can be traceably calibrated by striking a mass and measuring its displacement. 


\section{Conclusions}

The following are the primary conclusions from the activities described in this work:

(a) The design of the instrumented striker is of great importance for achieving low-uncertainty measurements via dynamic calibration. The sensitivity of the striker to impact location and angle affects the ability to calibrate it accurately, and the repeatability of test results. Having a large frequency range over which the striker response is nominally static will reduce the uncertainty achieved and can even eliminate the need for dynamic calibration for at least some Charpy tests. Minimizing nonlinearity of the striker response will allow low-uncertainty measurements based on a dynamic calibration.

(b) Of the two methods tested - the cut wire method and an impacting transfer transducer the transfer transducer method appears more promising given the faster testing rate and prospects for extending the calibrated bandwidth above $5 \mathrm{kHz}$. Mechanization of the transfer calibration impact is advantageous for minimizing the calibration uncertainty.

As described in section 5, work is underway to apply these lessons to the dynamic calibration of instrumented Charpy strikers.

\section{References}

[1] McCowan CN, Siewert TA, Vigliotti DP (2003) Charpy verification program: reports covering 1989-2002. NIST Technical Note 1500-9, Materials Reliability Series.

[2] Vlajic N, Chijioke A (2017) Impact mechanics parametric studies with applications to dynamic force calibration. ASME 2017 International Design Engineering Technical Conference \& Computers and Information in Engineering Conference, paper DETC201767893.

[3] Lucon E (2008) On the effectiveness of the dynamic force adjustment for reducing the scatter of instrumented Charpy results. Journal of ASTM International 6(1): 1-9.

[4] ASTM International, ASTM E2298-15, "Standard test method for instrumented impact testing of metallic materials,” https://doi.org/https://doi.org/10.1520/E2298-15.

[5] Ireland DR (1974) Procedures and Problems Associated with Reliable Control of the Instrumented Impact Test. Instrumented Impact Testing, ASTM STP 563, T. S. DeSisto, Ed., American Society for Testing and Materials, Philadelphia PA: 3-29. http://dx.doi.org/10.1520/STP32183S.

[6] Lucon E (2015) Determination of the compliance of NIST Charpy impact machines. NIST IR 8043, National Institute of Standards and Technology.

[7] Inoue H, Ishida H, Kishimoto K, Shibuya T (1991) Measurement of impact load by using an inverse analysis technique (comparison of methods for estimating the transfer function and its application to the instrumented Charpy impact test). JSME International Journal, Series I, 34: 453-457.

[8] Mackin T, Tognarelli D (1995) Design and evaluation of a verification system for force measurement using instrumented impact testing machines. Pendulum Impact Machines: Procedures and Specimens for Verification, ASTM STP 1248, T. Siewert and A. Schmieder, Ed., ASTM International, West Conshohocken, PA: 268-282. http://dx.doi.org/10.1520/STP14669S. 
[9] Riad SM (1986) The deconvolution problem: an overview. Proceedings of the IEEE 74, no. 1: 82-85. http://dx.doi.org/10.1109/PROC.1986.13407.

[10] "International vocabulary of metrology - Basic and general concepts and associated terms (VIM) - JCGM 200 V2, OIML V 2-200 and ISO/IEC Guide 99".

[11] Eichstädt S, Wilkens V, Dienstfrey A, Hale P, Hughes B, Jarvis C (2014) On challenges in the uncertainty evaluation for time-dependent measurements. Metrologia 53: S125.

[12] International Organization for Standardization, ISO 14556:2015, "Metallic materials - Charpy V-notch pendulum impact test -- Instrumented test method," Geneva (Switzerland). http://dx.doi.org/10.1088/0026-1394/53/4/S125.

[13] Lucon E, Scibetta M, McColskey JD, McCowan CN (2009) Influence of loading rate on the calibration of instrumented charpy strikers. Journal of Testing and Evaluation 37: 520-530.

[14] Manahan MP, Stonesifer RB (2000) The difference between total absorbed energy measured using an instrumented striker and that obtained using an optical encoder. Pendulum Impact Testing: A Century of Progress, ASTM STP 1380, T. Siewert and M. Manahan, Ed., ASTM International, West Conshohocken, PA: 181-197. http://dx.doi.org/10.1520/STP14394S.

[15] Mee DJ (2003) Dynamic calibration of force balances for impulse hypersonic facilities. Shock Waves, 12: 443-455. http://dx.doi.org/10.1007/s00193-003-0181-6.

[16] Vlajic N, Chijioke A (2017) Traceable calibration and demonstration of a portable dynamic force transfer standard. Metrologia 54: S83. http://dx.doi.org/10.1088/16817575/aa75da.

[17] Kalthoff J, Van Walle E, Wilde G (1996) Variations of the sensitivity of instrumented ISO/DIN and ASTM-tups and their influence on the determination of impact energies in tests with ductile steels. ESIS Publication 20: 25-36.

[18] Mangolds B (1964) Effect of mounting variables on accelerometer performance. The Shock and Vibration Bulletin, 33: 1-12.

[19] Schlegel C, Kieckenap G, Glöckner B, Buss A, Kumme R (2012) Traceable periodic force calibration. Metrologia 49: 224-235. http://dx.doi.org/10.1088/0026-1394/49/3/224.

[20] Vlajic N, Chijioke A (2016) Traceable dynamic calibration of force transducers by primary means. Metrologia 53: S136. http://dx.doi.org/10.1088/0026-1394/53/4/S136.

[21] Eichstädt S and Wilkens V (2017) Evaluation of uncertainty for regularized deconvolution: A case study in hydrophone measurements. The Journal of the Acoustical Society of America 141: 4155-4167. http://dx.doi.org/10.1121/1.4983827.

[22] See for example the specification sheet Model Number 086E80 ICP Impact Hammer, Revision: C, PCB Piezotronics, Depew (2015).

[23] Manahan MP, Stonesifer RB (2002) Studies toward optimum instrumented striker designs. European Structural Integrity Society, Vol. 30, Elsevier, 2002: 221-228. http://dx.doi.org/10.1016/S1566-1369(02)80024-3.

[24] Vlajic N, Chijioke A, Lucon E (2018) Design considerations to optimize Charpy instrumented strikers. Submitted to Journal of Testing and Evaluation (presently under review). 